\title{
1
}

\section{Theory of Rashba Torques}

\subsection{Introduction}

Magnetic systems lacking inversion symmetry display unique properties both in their ground state and out-of-equilibrium. Bulk non-centrosymmetric ferro- or antiferromagnets (such as $(\mathrm{Ga}, \mathrm{Mn}) \mathrm{As}, \mathrm{MnSi}$ etc.) as well as asymmetrically grown magnetic multilayers all exhibit some level of chiral magnetic textures (either under the form of skyrmion crystals, metastable isolated skyrmions, or homochiral Néel domain walls) and some sort of current-driven spin-orbit torques (either through inverse spin galvanic effect or spin Hall effect). Since the original prediction of current-induced spin torque in a Rashba spin-orbit coupled interface [1], the research on the interplay between various spin-orbit torques and chiral magnetic textures has resulted in outstanding experimental observations $[2,3,4,5,6,7]$ and is currently opening thrilling perspectives for novel spin devices $[8,9]$.

In this chapter, we focus our attention on the theory of current-driven Rashba torques in a number of magnetic systems. We first introduce the nature of spinorbit coupling in systems lacking inversion symmetry, then discuss the important features of the Rashba torque in ferromagnetic two dimensional electron gases. In the third section, we review recent theories of spin-orbit torques in other forms of noncentrosymmetric magnets (dilute magnetic semiconductors, antiferromagnets, topological insulators). We conclude this chapter by reviewing recent experimental results that support the emergence of Rashba torques in magnets lacking inversion symmetry.

\subsection{Spin-orbit coupling in systems lacking inversion symmetry}

Spin-orbit coupling locks the linear momentum of a carrier $\hat{\mathbf{p}}$ with its spin angular momentum $\hat{\boldsymbol{\sigma}}$, via the potential gradient of the environment (usually a crystal),

$$
\hat{H}_{\mathrm{so}}=\xi \hat{\boldsymbol{\sigma}} \cdot(\hat{\mathbf{p}} \times \nabla V) .
$$

In other words, spin-orbit coupling acts like a momentum-dependent magnetic field on the itinerant spin, such that $\hat{H}_{\mathrm{so}}=-\mu_{\mathrm{B}} \hat{\boldsymbol{\sigma}} \cdot \mathbf{B}_{\mathbf{k}}$. Since spin-orbit coupling preserves time-reversal symmetry, in crystals lacking spatial inversion symmetry the spin-orbit field $\mathbf{B}_{\mathbf{k}}$ must be odd in momentum, i.e. $\mathbf{B}_{-\mathbf{k}}=-\mathbf{B}_{\mathbf{k}}$.

The first example of such an odd-in-k spin-orbit coupling was proposed by Dresselhaus in $\mathrm{ZnS}$ crystal structure. In this crystal, the inversion symmetry is broken by the presence of a two-atomic motif in the unit cell. By applying the $\mathbf{k} \cdot \mathbf{p}$ theory around the $\Gamma$-point, the spin-orbit term becomes [10] 


\section{Theory of Rashba Torques}

$$
\hat{H}_{\mathrm{D}_{3}}=\beta_{3}\left(\left(k_{x}^{2}-k_{y}^{2}\right) k_{z} \hat{J}_{z}+\left(k_{z}^{2}-k_{x}^{2}\right) k_{y} \hat{J}_{y}+\left(k_{y}^{2}-k_{z}^{2}\right) k_{x} \hat{J}_{x}\right) .
$$

This Hamiltonian is linear in angular momentum operator $\hat{\mathbf{J}}$ and cubic in linear momentum k. If strain is applied along the (001) direction [11],

$$
\hat{H}_{\mathrm{D}_{1}}^{001} \approx \beta_{1}\left(k_{x} \hat{J}_{x}-k_{y} \hat{J}_{y}\right)
$$

where $\beta_{1}=\beta_{3}\left\langle k_{z}^{2}\right\rangle$. These terms are now linear in momentum $\mathbf{k}$, and enable several fascinating effects such as (inverse) spin galvanic effect and spin helix phenomena in semiconductors [12]. In the case of wurtzite crystal structures such as GaN, the elongated hexagonal structure results in a bulk Rashba-like spin-orbit coupling [13].

In pioneering works addressing the transport properties of a low-doped two-dimensional electron gas (2DEG), Vasko [14] and Bychkov and Rashba [15] proposed that the sharp asymmetric potential drop at the interfaces of the $2 \mathrm{DEG}, \boldsymbol{\nabla} V \approx \partial_{z} V \mathbf{z}$, results in a very simple form of spin-orbit coupling

$$
\hat{H}_{R}=\frac{\alpha_{\mathrm{R}}}{\hbar} \hat{\boldsymbol{\sigma}} \cdot(\hat{\mathbf{p}} \times \hat{\mathbf{z}}),
$$

where $\alpha_{\mathrm{R}} \approx \hbar^{2} \partial_{z} V / 4 m^{2} c^{2}$. In low-doped semiconductor quantum wells, where only a few bands are present around the $\Gamma$-point, the Rashba parameter can be calculated using the $\mathbf{k} \cdot \mathbf{p}$ theory (i.e. the envelope function approach [17]). In III-V semiconductors, it is related to the spin-orbit splitting $\Delta_{\text {so }}$ of the valence $p$ bands and the gap $E_{g}$ between the $s$ conduction and $p$ valence bands $[16,18]$,

$$
\alpha_{\mathrm{R}}=c\left\langle\partial_{z} U\right\rangle\left[\frac{1}{E_{g}^{2}}-\frac{1}{\left(E_{g}+\Delta_{\mathrm{so}}\right)^{2}}\right]
$$

where $c$ is a numerical constant, and $U$ is the total potential acting on the holes of the valence band. Since $U$ can be tuned by the gate voltage, the Rashba coupling can be electrically controlled in experiments $[19,20]$. Rashba spin-orbit coupling has also been recently confirmed at hetero-oxides interfaces such as $\mathrm{LaAlO}_{3} / \mathrm{SrTiO}_{3}[21,22,23]$. As a reference, the Rashba parameter of InAlAs/InGaAs and $\mathrm{LaAlO}_{3} / \mathrm{SrTiO}_{3}$ can be as large as $0.01-0.07 \mathrm{eV} \cdot \AA[24,21]$.

Rashba spin-orbit coupling has also been reported at the surface and interfaces of certain metals using Angle-Resolved PhotoEmission Spectroscopy (ARPES). These measurements have confirmed the existence of spin-split surface states in $\mathrm{Au}[25,26]$, Ag [27], Bi compounds [28, 29,30], Gd [31] or even metal-based quantum wells [32,33,

34]. Rashba-type spin-orbit splitting up to $3 \mathrm{eV}$. $\AA$ has been observed in $\mathrm{Bi} / \mathrm{Ag}$ surface alloy [29]. The physics underlying the emergence of a Rashba-type spin splitting at metallic surfaces and interfaces is still under intense investigation but recent progress has shed some light on this problem. Several authors recently pointed out that the phenomenological picture stating that Rashba parameter is controlled by interfacial potential drop fails to quantitatively (even qualitatively) account for the experimental observations. Indeed, the Rashba spin splitting induced by the interfacial drop [i.e. the Rashba Hamiltonian given in Eq. (1.4)] is at least two orders of magnitude too small compared to experimental observations. Actually, while the spin-orbit coupling itself 
remains mostly unperturbed by the interfacial symmetry breaking, the wavefunction around the nucleus is strongly distorted $[35,36,37]$. In other words, the redistribution of the charge density close to the nucleus determines the sign and strength of the splitting [31,38]. The orbital character ( $p$ orbitals in Bi compounds, $d$ orbitals in transition metals) is therefore crucial to determine the strength and sign of the Rashba parameter at metallic surfaces and depends on the band index [39]. The existence of Rashba-type spin-splitting at normal metal/ferromagnet interfaces has been recently confirmed by ab initio calculations [39, 40,41].

Another class of systems that presents a very strong spin-momentum locking is the conductive surface of topological insulators $[42,43,44,45]$. Such materials are insulating in their bulk and possess topologically protected conducting surfaces, where spin angular momentum is locked to the linear momentum. In their simplest version, the Hamiltonian of the surface reads

$$
\hat{H}_{\mathrm{D}}=v \hat{\boldsymbol{\sigma}} \cdot(\hat{\mathbf{p}} \times \hat{\mathbf{z}}),
$$

which strikingly resembles the Rashba Hamiltonian. The equivalent Rashba parameter ranges from $\sim 2 \mathrm{eV} . \AA$ for $\mathrm{Bi}_{2} \mathrm{Te}_{3}$ [46] to $4.1 \mathrm{eV} . \AA$ for $\mathrm{Bi}_{2} \mathrm{Se}_{3}$ [47] and $5.7 \mathrm{eV} . \AA$ for Kawazulite [i.e. $\mathrm{Bi} 2(\mathrm{Te}, \mathrm{Se}) 2(\mathrm{Se}, \mathrm{S})][48]$.

\subsection{Rashba torques in magnetic two dimensional electron gas}

\subsubsection{Free electron model}

A convenient model to investigate the current-driven spin-orbit torques in an inversion symmetry broken ferromagnet is the magnetic 2DEG in the presence of Rashba spinorbit coupling. The model Hamiltonian reads

$$
\hat{H}=\frac{\hat{\mathbf{p}}^{2}}{2 m}-\frac{\alpha_{\mathrm{R}}}{\hbar} \hat{\boldsymbol{\sigma}} \cdot(\mathbf{z} \times \hat{\mathbf{p}})+\frac{\Delta}{2} \hat{\boldsymbol{\sigma}} \cdot \mathbf{m}
$$

where the first term is the kinetic energy, the second term is Rashba spin-orbit coupling and the last term accounts for the coupling between itinerant electrons spin $\hat{\boldsymbol{\sigma}}$ ( $s p$-like) and the local magnetization $\mathbf{m}$ (from $d$-like electrons - here $|\mathbf{m}|=1$ ). The eigenstates of this Hamiltonian are

$$
\begin{aligned}
& |+\rangle=\left(\begin{array}{c}
e^{i \gamma_{\mathbf{k}}} \cos \frac{\chi_{\mathbf{k}}}{2} \\
\sin \frac{\chi_{\mathbf{k}}}{2}
\end{array}\right),|-\rangle=\left(\begin{array}{c}
-e^{i \gamma_{\mathbf{k}}} \sin \frac{\chi_{\mathbf{k}}}{2} \\
\cos \frac{\chi_{\mathbf{k}}}{2}
\end{array}\right), \\
& \epsilon_{\mathbf{k}}^{s}=\frac{\hbar^{2} k^{2}}{2 m}+s \sqrt{\frac{\Delta^{2}}{4}+\alpha_{\mathrm{R}}^{2} k^{2}+\alpha_{\mathrm{R}} k \Delta \sin \left(\varphi_{\mathbf{k}}-\varphi\right) \sin \theta} .
\end{aligned}
$$

where

$$
\begin{aligned}
\cos \chi_{\mathbf{k}} & =\frac{\Delta \cos \theta}{\sqrt{\Delta^{2}+4 \alpha_{\mathrm{R}}^{2} k^{2}+4 \Delta \alpha_{\mathrm{R}} \sin \left(\varphi_{\mathbf{k}}-\varphi\right) \sin \theta}}, \\
\tan \gamma_{\mathbf{k}} & =\frac{2 \alpha_{\mathrm{R}} k \cos \varphi_{\mathbf{k}}-\Delta \sin \varphi \sin \theta}{2 \alpha_{\mathrm{R}} k \sin \varphi_{\mathbf{k}}+\Delta \cos \varphi \sin \theta}
\end{aligned}
$$

The eigenstates, Eq. (1.8), are helical in spin space and the interplay between Rashba spin-orbit coupling and $s-d$ exchange results in a distortion of the energy dispersion 


\section{Theory of Rashba Torques}

(and henceforth, of the Fermi surface) depending on the magnetization direction [see Eq. (1.9)]. For instance, if one considers a perpendicularly magnetized ferromagnet $(\theta=0)$, the spin density of state $s(= \pm)$ is

$$
\mathbf{s}_{\mathbf{k}}^{s}=-s \frac{2 \alpha_{\mathrm{R}}}{\sqrt{\Delta^{2}+4 \alpha_{\mathrm{R}}^{2} k^{2}}} \mathbf{z} \times \mathbf{k}+s \frac{\Delta}{\sqrt{\Delta^{2}+4 \alpha_{\mathrm{R}}^{2} k^{2}}} \mathbf{z} .
$$

\subsubsection{Current-induced Rashba spin torques}

The above simple solution of the momentum-dependent spin density, Eq. (1.12), does not generate a net magnetic moment in equilibrium since the summation of all $\mathbf{k}$ is zero. However, when a current is applied, a net magnetic moment appears. In a nutshell, $\langle\mathbf{k}\rangle \sim \mathbf{j}$, and from Eq. (1.12) the non-equilibrium spin accumulation reads $\mathbf{S} \propto \mathbf{z} \times \mathbf{j}$, an effect called inverse spin galvanic effect [49] or Rashba-Edelstein effect [50]. In 2008, Manchon and Zhang proposed that such current-induced spin accumulation could yield a novel spin torque on the contacting magnetic layers [1]: First, the spin accumulation serves as an effective magnetic field through the direct exchange coupling with the ferromagnetic magnetization $\mathbf{m}$ and thus

$$
\mathbf{T}_{\mathrm{FL}}=\Delta \mathbf{m} \times \mathbf{S} \propto \mathbf{m} \times(\mathbf{z} \times \mathbf{j}) .
$$

This term is known as the field-like SOT. Second, the interface spin accumulation can diffuse into the ferromagnetic layer and subsequently being absorbed; this process is known as the spin-transfer torque (or Slonczewski-spin torque, or damping-like torque) which could be simply expressed by

$$
\mathbf{T}_{\mathrm{DL}}=G^{\uparrow \downarrow} \mathbf{m} \times(\mathbf{S} \times \mathbf{m}) \propto \mathbf{m} \times[(\mathbf{z} \times \mathbf{j}) \times \mathbf{m}],
$$

where $G^{\uparrow \downarrow}$ is the spin conductivity of the interface (or mixing conductance), $\mathbf{S}$ is the interfacial spin accumulation. We point out that the damping-like torque could also come from another competing mechanism known as the spin Hall torque: a spin current generated by the spin Hall effect [71] could produce the mathematically identical forms of the SOT $[40,72]$. Sometimes, the experimentally observed damping-like torque has been attributed to the spin Hall effect; this is an erroneous assertion since the interface Rashba effect alone could also generate the damping-like torque. To determine the relative contributions from the bulk spin Hall and interface Rashba for the dampinglike torque, geometrical (e.g., thickness) and material dependence of the spin torque should be analyzed in detail.

Since the intial prediction of the above Rashba torques and subsequently verified experimentally $[2,51]$, much studies on the SOT have been carried out in the past eight years in both homogeneous metallic ferromagnets $[52,53,54,55,56,57,58,59,60]$ and magnetic textures $[61,62,63,64,65,66,67,68,69,70]$. In the following, we discuss an improved calculation of the Rashba spin torques.

Boltzmann transport equation $[1,52,53,55,59]$, or quantum kinetics $[56,57,58,60]$ have been used to compute the non-equilibrium property. Although formally equivalent, these methods involve different levels of approximations (relaxation time approximation, first Born approximation, vertex corrections etc.) resulting in quantitative 
differences between the computed Rashba torques. For instance, using Kubo formalism one can compute the non-equilibrium spin density assuming spin-independent scattering rate and in the limit of weak $\left(\alpha_{\mathrm{R}} k_{\mathrm{F}} \gg \Delta\right)$ and strong ferromagnetism $\left(\Delta \gg \alpha_{\mathrm{R}} k_{\mathrm{F}}\right)[58]$

$$
\begin{aligned}
& \mathbf{T}_{\alpha_{\mathrm{R}} k_{\mathrm{F}} \gg \Delta} \approx \frac{\alpha_{\mathrm{R}} n_{\mathrm{F}}}{4 \Gamma} \frac{\Delta}{\epsilon_{\mathrm{F}}}\left(\mathbf{m} \times(\mathbf{z} \times e \mathbf{E})+\frac{\Delta \Gamma}{\alpha_{\mathrm{R}}^{2} k_{\mathrm{F}}^{2}} m_{z} \mathbf{m} \times(\mathbf{z} \times e \mathbf{E})\right), \\
& \mathbf{T}_{\Delta \gg \alpha_{\mathrm{R}} k_{\mathrm{F}}} \approx \frac{\alpha_{\mathrm{R}} n_{\mathrm{F}}}{2 \Gamma} \frac{\Delta}{\epsilon_{\mathrm{F}}}\left(\mathbf{m} \times(\mathbf{z} \times e \mathbf{E})-\frac{2 \Gamma}{\Delta} \mathbf{m} \times[(\mathbf{z} \times e \mathbf{E}) \times \mathbf{m}]\right) .
\end{aligned}
$$

Here $\Gamma$ is the (spin-independent) impurity broadening, $n_{\mathrm{F}}$ is the total electron density and $\epsilon_{\mathrm{F}}$ is Fermi energy. A similar form was derived by various authors using different approximations $[55,56,57,59,60]$. The fact that the torque is composed of two components, one odd in magnetization $\propto \mathbf{m} \times(\mathbf{z} \times \mathbf{E})$ and one even in magnetization $\propto \mathbf{m} \times[(\mathbf{z} \times \mathbf{E}) \times \mathbf{m}]$ is a general feature of SOTs, not limited to Rashba gases. The field-like torque, $\propto \mathbf{m} \times(\mathbf{z} \times \mathbf{E})$, is simply the consequence of inverse spin galvanic effect explained above (i.e. Rashba-Edelstein effect) $[1,50]$. The origin of the damping-like torque, $\propto \mathbf{m} \times[(\mathbf{z} \times \mathbf{E}) \times \mathbf{m}]$, is more subtle. Two main origins have been identified. First, as mentioned above, spin dephasing and relaxation distorts the spin dynamics in the two dimensional gas, resulting in a correction to the inverse spin galvanic effect. This general principle has been originally pointed out in the case of spin-valves [73] and ferromagnetic domain walls [74], and applies consistently to systems with broken inversion symmetry. The second origin of the damping-like torque is related to the Berry curvature of the electronic band structure in the mixed spin-momentum phase space $[55,58,59,75,76]$. This contribution does not vanish in the limit of weak disorder and is quite sensitive to "hot spots" in the band structure, i.e. points where neighboring bands get very close to each other and where contributions of the form $\sim 1 /\left(\epsilon_{n}-\epsilon_{n^{\prime}}\right)^{2}$ becomes very large [58]. We emphasize that the results discussed above in the context of the Rashba model have been qualitatively confirmed by ab initio calculations on realistic transition metal interfaces $[40,76,77]$.

We conclude this section by mentioning the diffusive spin dynamics in a magnetic Rashba gas. In the limit of strong disorder $\left(\epsilon_{\mathrm{F}} \gg \Gamma \gg \Delta, \alpha_{\mathrm{R}} k_{\mathrm{F}}\right)$, the spin-charge coupled drift-diffusion equations in the two dimensional magnetic Rashba gas read [56]

$$
\begin{aligned}
\frac{\partial n}{\partial t}= & \mathcal{D} \nabla^{2} n+\mathcal{K}_{\mathrm{sc}}(\mathbf{z} \times \boldsymbol{\nabla}) \cdot \mathbf{S}+\mathcal{R} \nabla_{z} \cdot \mathbf{m}(\mathbf{S} \cdot \mathbf{m})+\mathcal{G}(\mathbf{z} \times \boldsymbol{\nabla}) \cdot \mathbf{m} n \\
\frac{\partial \mathbf{S}}{\partial t}= & \mathcal{D} \nabla^{2} \mathbf{S}-\frac{\mathbf{S}}{\tau_{\mathrm{sf}}}-\frac{S_{z} \mathbf{z}}{\tau_{\mathrm{z}}}-\frac{1}{\tau_{\Delta}} \mathbf{S} \times \mathbf{m}-\frac{1}{\tau_{\varphi}} \mathbf{m} \times(\mathbf{S} \times \mathbf{m}) \\
& +\mathcal{K}_{\mathrm{sc}}(\mathbf{z} \times \boldsymbol{\nabla}) n+2 \mathcal{K}_{p} \boldsymbol{\nabla}_{z} \times \mathbf{S} \\
& +\mathcal{G}[\mathbf{m} \times((\mathbf{z} \times \boldsymbol{\nabla}) \times \mathbf{S})+(\mathbf{z} \times \boldsymbol{\nabla}) \times(\mathbf{m} \times \mathbf{S})]+2 \mathcal{R}[\mathbf{m} \cdot(\mathbf{z} \times \boldsymbol{\nabla})] n \mathbf{m} .
\end{aligned}
$$

Let us comment these equations briefly. The first equation, Eq. (1.17), concerns the diffusion of the charge density $n$, driven by the spin-charge coupling $\sim \mathcal{K}_{\mathrm{sc}}$ : a gradient in spin density $(\mathbf{z} \times \boldsymbol{\nabla}) \cdot \mathbf{S}$ produces a change in the charge density. Physically, this equation states that a spatial gradient in spin density produces a charge current: this is the spin-injection Hall effect [78]. The two additional terms, $\sim \mathcal{G}$ and $\sim \mathcal{R}$, are 
higher order corrections that account for the precession of the non-equilibrium spin density $\mathbf{S}$ around the local magnetization $\mathbf{m}$. The second equation, Eq. (1.18), describes the spin dynamics induced by a charge gradient (i.e. an electric field). The spin dynamics involves anisotropic spin relaxation, $\sim 1 / \tau_{\text {sf }}$ and $\sim 1 / \tau_{\mathrm{z}}$ (i.e. the well-known D'yakonov-Perel spin relaxation in Rashba gases [79]), the spin precession around the magnetization $\sim 1 / \tau_{\Delta}$ and some spin dephasing $\sim 1 / \tau_{\varphi}$. In addition, spin-charge conversion processes take place, such as the spin Hall effect $\sim \mathcal{K}_{\mathrm{sc}}[71]$ and the inverse spin galvanic effect $\sim \mathcal{K}_{\mathrm{p}}[49]$. The other terms are higher order corrections. The competition between these different terms give rise to a fairly complex spin dynamics, and most importantly to a torque that possesses both field-like and damping-like components, as discussed above and consistently with Kubo formula derivations [56].

\subsubsection{Rashba torque in magnetic textures}

Besides the investigation of Rashba torque in homogeneous ferromagnets, the physics of SOT-driven magnetic domain wall has also attracted substantial interest $[5,6]$. The impact of Rashba torque on the dynamics of domain walls has been initially studied by Obata and Tatara [61]. The authors showed that the Rashba field promotes the motion of in-plane Bloch walls whose hard axis is oriented along the current direction. Such a prediction has been followed by several publications focusing on the dynamics of domain walls under both field-like and damping-like SOTs $[62,63,66,67,64,65,68$, $69,70]$.

For instance, it was shown that Rashba field-like torque can stabilize perpendicularly magnetized Bloch walls, thereby suppressing the Walker breakdown and resulting in gigantic domain wall velocities [5] (see also [63, 69]). Kim et al. [80, 81] also showed that Rashba spin-orbit coupling enables charge pumping, enhances the magnetic damping and can mediate antisymmetric Dzyaloshinskii-Moriya interaction. The latter interaction turns out to be a crucial ingredient of non-centrosymmetric magnets. One of the most important outcomes of these studies has been the prediction that the combination between Dzyaloshinskii-Moriya interaction (that stabilizes homochiral Néel walls) and damping-like SOT (that drives the motion of such walls) leads to extremely fast domain wall velocities [82], of highest importance for applications [6].

\subsection{Beyond the magnetic two dimensional electron gas}

\subsubsection{Bulk dilute magnetic semiconductors}

III-V dilute magnetic semiconductors such as $(\mathrm{Ga}, \mathrm{Mn})$ As present an interesting paradigm for SOTs as they display large bulk spin-orbit coupling together with inversion asymmetry. As such, they constitute the first class of systems in which SOTs have been observed $[51,83,84]$. The theory of current-driven torques in dilute magnetic semiconductors was first studied by Bernevig and Vafek [85]. The authors considered the Kohn-Luttinger Hamiltonian in the spherical approximation with an exchange energy, augmented by a spin-orbit coupling term of the form $\boldsymbol{\lambda}(\mathbf{k}) \cdot \hat{\mathbf{J}}$ where $\lambda_{x}(\mathbf{k})=$ $C_{4}\left(\epsilon_{x y} k_{y}-\epsilon_{x z} k_{z}\right)$ and $\lambda_{y, z}$ are obtained from cyclic permutation of indices. The current-driven spin density reads 


$$
\mathbf{S}=\langle\hat{\mathbf{J}}\rangle=-\left(\frac{3 n}{\pi}\right)^{1 / 3} \frac{\tau}{\hbar^{3}} \frac{15}{2} \frac{m}{\gamma_{1}}\left(\sum_{s= \pm 1} \frac{1}{\left(1+2 s \gamma_{2} / \gamma_{1}\right)^{3 / 2}}\right)^{2 / 3}\left(e \mathbf{E} \cdot \nabla_{\mathbf{k}}\right) \boldsymbol{\lambda}
$$

where $\gamma_{1,2}$ are the Luttinger parameters defining the band structure, $\tau$ is the momentum relaxation time, $n$ is the charge density and $\mathbf{E}$ is the applied electric field. The torque induced by this inverse spin galvanic effect is therefore a field-like torque. A similar torque has been numerically computed in [86,87]. A few years later, combining both theory and experiments, Kurebayashi et al. [75] proposed that intrinsic contributions could result in damping-like torque. These contributions were not considered in previous works $[85,86,87]$ and have been discussed extensively recently [58]. It is quite interesting to notice that the torques computed in dilute magnetic semiconductors exhibit properties qualitatively similar to the ones featured by SOTs in the magnetic Rashba gas.

\subsubsection{Dirac Torques in topological insulators}

Three dimensional topological insulators are a new class of materials that have an insulating bulk and spin-momentum-locked metallic surface states $[43,108]$. They exhibit strong spin-orbit coupling and are expected to show large charge-to-spin current conversion efficiency, as reported by recent experiments [109,110,111,112]. A wide disparity exists between the various results and the physics behind spin-charge conversion at the surface of topological insulators is still a matter of debate: the nature of interfacial states and the importance of spin Hall effect remain essentially unknown.

In its simplest version, the low-energy Hamiltonian of the surface of a topological insulator reads

$$
\hat{H}_{0}=v \hat{\boldsymbol{\sigma}} \cdot(\hat{\mathbf{p}} \times \mathbf{z})+\frac{\Delta}{2} \hat{\boldsymbol{\sigma}} \cdot \mathbf{m}
$$

where $v$ is the Fermi velocity, and $\Delta$ is the exchange coupling between the spin and the magnetization m. This Hamiltonian looks very similar to the Rashba Hamiltonian, Eq. (1.7), in the absence of kinetic term. Two remarks are in order though. First, since the kinetic term is solely given by the Dirac coupling, $\sim \hat{\boldsymbol{\sigma}} \cdot(\hat{\mathbf{p}} \times \mathbf{z})$, the spin density and charge current density are directly proportional to each other, $\mathbf{j} \sim \mathbf{z} \times \mathbf{S}$. In other words and quite unsurprisingly, the Dirac term produces inverse spin galvanic effect, similar to the case of Rashba. Second, and most importantly, the in-plane magnetization $m_{x}, m_{y}$ can be removed from Eq. (1.20) by a simple gauge transformation. This means that only the magnetization component normal to the surface, $\sim m_{z}$, impacts the transport properties of the topological insulator.

As a matter of fact, a perpendicular magnetization opens a gap in the Dirac cone, thereby driving a topological phase transition [113]. When the Fermi energy lies in the gap and the system is in the insulating regime, a magnetic topological insulator exhibits two effects that are the hallmark of three dimensional topological insulators: quantum anomalous Hall effect [115], and quantum magnetoelectric effect [113, 114]. The former is the emergence of a quantized anomalous Hall conductance, $\sigma_{\mathrm{H}}=\mathcal{C} e^{2} / h$ ( $\mathcal{C}$ being the Chern number characterizing the topological insulator), while the latter is the emergence of a quantized spin density aligned along the electric field direction, $\mathbf{S}=-\left(\sigma_{\mathrm{H}} / e v\right) \mathbf{E}[114]$. Because of the gap opening, magnetic domain walls or magnetic 


\section{Theory of Rashba Torques}

vortices are accompanied by electric charging, which has a direct impact on their dynamics $[116,118,117]$.

The nature of SOT in the metallic regime has been the object of numerous theoretical investigations $[121,122,119,120,123,124,125,126]$. The electric field-induced SOT reads [127]

$$
\mathbf{T}=-\frac{\hbar v n_{\mathrm{F}}}{4 \Gamma} \frac{\Delta}{\epsilon_{\mathrm{F}}} \frac{1-\beta^{2} m_{z}^{2}}{1+3 \beta^{2} m_{z}^{2}}\left(\mathbf{m} \times(\mathbf{z} \times e \mathbf{E})+\frac{4 \Delta \Gamma}{\epsilon_{\mathrm{F}}^{2}} \frac{1+\beta^{2} m_{z}^{2}}{1-\beta^{2} m_{z}^{2}} \frac{1}{1+3 \beta^{2} m_{z}^{2}} m_{z} \mathbf{m} \times e \mathbf{E}\right) .
$$

Here $\beta=\Delta / 2 \epsilon_{\mathrm{F}}$ is the polarization and the calculation assumes $\epsilon_{\mathrm{F}}>\Delta / 2$. The first term $\sim \mathbf{m} \times(\mathbf{z} \times e \mathbf{E})$ is simply the field-like torque due to inverse spin galvanic effect, while the second term is the damping-like torque due to magnetoelectric effect. This structure is actually very similar to the Rashba torque given in Eq. (1.15), i.e. in the large Rashba limit, although of opposite sign [120]. Interestingly, the dampinglike torque vanishes when the magnetization lies in the surface plane. Furthermore, a strong angular dependence is expected. We note that a strong, but opposite angular dependence of the torque has been experimentally identified in magnetically-doped topological insulators [110]: in this experiment the magnitude of the torque is larger when the magnetization lies perpendicular to the plane of the surface. In other words, the toy model presented above, Eq. (1.20), is insufficient to explain the experimental data.

\subsubsection{Antiferromagnetic two dimensional electron gas}

Up till recently, antiferromagnets were mainly studied under two different perspectives, one fairly applied, the exchange bias [88], and one quite fundamental, the nature of certain exotic states [89]. In 2006, Nuñez et al. [90,91] suggested that spin transfer torque could be achieved in a spin-valve device composed of two antiferromagnetic electrodes. In the course of the search for such spin transfer torques, it was realized that in order to control the order parameter of a collinear bipartite antiferromagnet, one needs a torque that is even in magnetization, in other words a damping-like torque [92]. Such a damping-like torque can be obtained by several ways, using a ferromagnetic polarizer [93], spin Hall effect [94, 95] or SOTs [96, 97]. The recent demonstration of current-driven SOT in CuMnAs [7] has opened thrilling avenues for the development of this field $[98,99]$.

The SOT in antiferromagnet can be computed analytically in a toy model consisting of a two dimensional electron gas with both Rashba spin-orbit coupling and antiferromagnetism. In the present case, we consider a G-type antiferromagnet with nearest neighbor hopping only, i.e. each magnetic site $A$ is surrounded by sites $B$ whose magnetic moment is aligned antiparallel. The low energy Hamiltonian of such a system reads [96]

$$
\tilde{H}=\gamma_{k} \hat{\tau}_{x}-\frac{\alpha_{\mathrm{R}}}{\hbar} \hat{\boldsymbol{\sigma}} \cdot(\mathbf{z} \times \hat{\mathbf{p}}) \hat{\tau}_{x}+\frac{\Delta}{2} \mathbf{n} \cdot \hat{\boldsymbol{\sigma}} \hat{\tau}_{z},
$$

where $\gamma_{k}=\left(\hbar^{2} / 2 m\right)\left(k^{2}-k_{0}^{2}\right)$. Here, $\hat{\boldsymbol{\sigma}}$ and $\hat{\boldsymbol{\tau}}$ are Pauli spin operators describing the real spin $\{|\uparrow\rangle,|\downarrow\rangle\}$ and the sublattice $\{|\mathrm{A}\rangle,|\mathrm{B}\rangle\}$ spaces, respectively, and $\mathbf{n}$ is the 
Néel order parameter. In the case where the magnetic order parameter $\mathbf{n}$ is normal to the plane $(\mathbf{n} \approx \mathbf{z})$, one can determine the Rashba torque on sublattice $i$ analytically ( $i= \pm 1$ for A and B sublattices, respectively),

$$
\mathbf{T}_{i}=\frac{\alpha_{\mathrm{R}} n_{\mathrm{F}}}{4 \Gamma} \frac{\Delta}{\epsilon_{\mathrm{F}}}\left((-1)^{i} \mathbf{n} \times(\mathbf{z} \times e \mathbf{E})+\frac{\Delta \Gamma}{\epsilon_{\mathrm{F}}^{2}} \mathbf{n} \times[(\mathbf{z} \times e \mathbf{E}) \times \mathbf{n}]\right) .
$$

This expression has been derived to the lowest order of $\alpha_{\mathrm{R}}\left(\Delta \gg \alpha k_{\mathrm{F}}\right)$, close to the extremum of the band (top or bottom), and assuming a spin-independent impurity broadening $\Gamma$.

One can compare these formulae with Eq. (1.16). The structure of the torque is quite similar to the one obtained in the ferromagnetic Rashba gas. The first term, $\sim(-1)^{i} \mathbf{n} \times(\mathbf{z} \times e \mathbf{E})$, is a torque that changes sign on opposite sublattices, i.e. it is simply a field-like torque, as expected from inverse spin galvanic effect. In other words, it cannot torque the antiferromagnetic order parameter. The second term has the same sign on opposite sublattices and is therefore associated with a staggered field [96, 97]. This one can efficiently torque the antiferromagnetic order parameter.

\subsubsection{Two dimensional hexagonal lattices}

The theoretical investigation of inverse spin galvanic effect and SOTs has been recently extended towards two dimensional hexagonal lattices such as, but not limited to, graphene, silicene, germanene, stanene, transition metal dichalcogenides etc. $[100,101,102]$. The parametric dependence of the torque in these materials does not significantly differ from the toy model of the Rashba two dimensional electron gas or from the richer three dimensional dilute magnetic semiconductors discussed above. A few noticeable aspects are worth noticing though. First of all, the charge transport in two dimensional hexagonal lattices is driven by two independent valleys. As long as intervalley scattering remains weak, one can generate two torques of different magnitudes on each valley. Second, such two dimensional hexagonal lattices are handy platforms to explore topological phase transition between normal metals to band insulators and quantum (spin, valley, anomalous) Hall regimes.

\subsubsection{Semi-magnetic tunnel junctions}

Let us conclude this section by addressing a last interesting setup. Consider a magnetic tunnel junction composed of a ferromagnet and a normal metal separated by a tunnel barrier. In this setup, the current is injected perpendicular to the plane of the layer, i.e. through the tunnel barrier. If spin-orbit coupling is strong in the ferromagnet, such a system exhibits tunneling anisotropic magnetoresistance: although only one ferromagnet is involved, the overall resistance of the device depends on the orientation of the magnetization with respect to the crystallographic directions of the system $[103,104,105]$. A minimal model to describe tunneling anisotropic magnetore-

sistance assumes some form of Rashba spin-orbit coupling at the interface between the ferromagnet and the barrier. One can show easily that such a spin-orbit coupling also enables SOT on the ferromagnet $[106,107]$. Following the symmetry of the system, the torque is on the form $\mathbf{T} \sim \mathbf{m} \times \mathbf{z}+\mathbf{m} \times(\mathbf{z} \times \mathbf{m})$, where $\mathbf{z}$ is the normal to the interface. 


\subsection{Experimental evidence of Rashba torques}

\subsubsection{Transition metal interfaces}

SOTs have been massively studied in asymmetric transition metal multilayers $[3,4,6]$ (such as $\mathrm{Pt} / \mathrm{Co} / \mathrm{AlOx}, \mathrm{Ta} / \mathrm{NiFe} / \mathrm{MgO}$ etc.) and we do not intend to review all these important works here (see e.g. [128]). In general, the SOTs observed in these systems adopt the form $\mathbf{T} \sim \mathbf{m} \times(\mathbf{z} \times \mathbf{E})+\mathbf{m} \times[(\mathbf{z} \times \mathbf{E}) \times \mathbf{m}]$, which is in principle consistent with the Rashba torque described in the previous sections. Although it is clear that very large Rashba spin-orbit coupling exists at heavy metal surfaces [41], it remains very difficult to accurately determine the origin of the SOT as spin Hall effect is usually present in these systems.

One way to obtain Rashba torques is to consider a system where spin Hall effect is absent or vanishingly small. In our original theory [1], we suggested that Rashba torque at the interface between $\mathrm{Co}$ and $\mathrm{AlOx}$ could be sizable. The physical picture behind this idea was elaborated based on experiments on perpendicular magnetic anisotropy at $\mathrm{Co} / \mathrm{AlOx}$ interfaces $[129,130]$. These experiments demonstrated that upon varying the oxidation of the AlOx layer, one could significantly enhance the perpendicular magnetic anisotropy, and as we though at that time, the Rashba spin-orbit coupling. Although quite naive in regard to the recent developments of the field, this initial guess has been confirmed by two experiments. Recently, Emori et al. [131] investigated the nature of the SOT a thin NiFe film sandwiched between Ti and AlOx. Here, Ti has a small spin-orbit coupling and spin Hall effect is expected to be vanishingly small. The authors obtained a field-like torque significantly larger than the Oersted field that they attributed to the Rashba effect at $\mathrm{NiFe} / \mathrm{AlOx}$ interface. In another work, Qiu et al. [132] reported that tuning the amount of oxygen at the $\mathrm{CoFeB} / \mathrm{MgO}$ interface could lead to a complete change of sign of SOT, thereby demonstrating that Rashba torque as $\mathrm{CoFeB} / \mathrm{MgO}$ interface can be quite significant.

\subsubsection{Non-centrosymmetric magnets}

Bulk non-centrosymmetric magnets constitute the first class of materials in which SOT has been predicted $[85,86,87]$ and observed $[51,83,84]$. They also present a unique platform for the investigation of SOTs: since no spin Hall effect is present, SOT entirely comes from the bulk of the materials, i.e. from inverse spin galvanic effect. The exact form of the torque can be deduced from the crystal symmetries of the magnet following Neumann's principle $[133,97]$. This has been recently extended to NiMnSb [134] and CuMnAs [7], as discussed above.

\subsubsection{Oxide heterostructures}

Oxide heterostructures such as $\mathrm{LaAlO}_{3} / \mathrm{SrTiO}_{3}$ has attracted a significant amount of attention in the past ten years due to their ability to support high-mobility two dimensional electron gases $[135,136,137]$. Most interestingly for spintronics applications, these structures exhibit both sizable magnetism [138,139,140], efficient spin injection [141,142], as well as large Rashba spin-orbit coupling [21,22,23]. Although, to the best of our knowledge, no SOTs experiments have been performed to date, room temperature measurement of the Rashba field has been recently reported. By probing 
the anisotropic magnetoresistance of $\mathrm{LaAlO}_{3} / \mathrm{SrTiO}_{3}$ superlattices, Narayanapillai et al. [143] extracted a Rashba field as large as 2 Telsa.

\subsection{Conclusion}

The electrical control of small magnetic objects mediated by spin-orbit coupling has driven a lot of excitation in the spintronics community. Among the most fascinating directions pursued nowadays, antiferromagnets, topological materials and potentially oxide interfaces seem to carry the most promising perspectives. The present chapter focused on one aspect only, the current-driven Rashba torque, and disregarded the spin Hall torque. As mentioned in this chapter, distinguishing between Rashba and spin Hall torques remains a matter of debate and an extensive discussion is out of the scope of the present review. The development of novel materials with strong spin-orbit coupling as well as the realization of room temperature non-trivial magnetic topologies (such as, but not limited to, magnetic skyrmions) will undoubtedly maintain a very high level of interest in this extremely rich field. 


\section{References}

[1] A. Manchon and S. Zhang, Phys. Rev. B 78, 212405 (2008).

[2] I. M. Miron, G. Gaudin, S. Auffret, B. Rodmacq, A. Schuhl, S. Pizzini, J. Vogel and P. Gambardella, Nature Materials 9, 230 (2010).

[3] I. M. Miron, K. Garello, G. Gaudin, P.-J. Zermatten, M. V. Costache, S. Auffret, S. Bandiera, B. Rodmacq, A. Schuhland, and P. Gambardella, Nature 476, 189193 (2011).

[4] L. Liu, C.-F. Pai, Y. Li, H. W. Tseng, D. C. Ralph, and R. A. Buhrman, Science 336, 555 (2012).

[5] I.M.Miron, T.Moore, H.Szambolics, L.D.Buda-Prejbeanu, S.Auffret, B.Rodmacq, S.Pizzini, J. Vogel, M.Bonfim, A.Schuhl, G.Gaudin, Nat.Mat.10, 419 (2011).

[6] S.H. Yang, K.S. Ryu and S. Parkin, Nat. Nanotechnology 10, 221 (2015).

[7] P. Wadley, B. Howells, J. Železný, C. Andrews, V. Hills, R. P. Campion, V. Novák, F. Freimuth, Y. Mokrousov, A. W. Rushforth, K. W. Edmonds, B. L. Gallagher, and T. Jungwirth, Science 351, 587-590 (2016).

[8] L. Liu, C.-F. Pai, D. C. Ralph, and R. A. Buhrman, Phys. Rev. Lett. 109, 186602 (2012).

[9] K. Garello, C. O. Avci, I. M. Miron, M. Baumgartner, A. Ghosh, S. Auffret, O. Boulle, G. Gaudin, and P. Gambardella, Appl. Phys. Lett. 105, 212402 (2014).

[10] G. Dresselhaus, Phys. Rev. 100, 580 (1955).

[11] M.I. Dyakonov, V.A. Marushchak, V. I. Perel, and A.N. Titkov Zh. Eksp. Teor. Fiz 90, 1123 (1986).

[12] A. Manchon, H. C. Koo, J. Nitta, S. M. Frolov, and R. A. Duine, Nature Materials 14, 871 (2015).

[13] E. Rashba, Sov. Phys. Solid State 2, 1109-1122 (1960).

[14] F.T. Vas?ko, P. Zh. Eksp. Teor. Fiz. 30, $574 ? 577$ (1979).

[15] Y.A. Bychkov, and E.I. Rasbha, P. Zh. Eksp. Teor. Fiz. 39, 66-69 (1984).

[16] R. Winkler, Spin-Orbit Coupling effects in Two-Dimensional Electron and Hole Systems (Springer, 2003).

[17] R. Lassnig, Phys. Rev. B 31, 8076 (1985).

[18] Th. Schapers, G. Engels, J. Lange, M. Hollfelder, and H. Luth, J. Appl. Phys. 83, 4324-4333 (1998).

[19] J. Nitta, T. Akazaki, H. Takayanagi, and T. Enoki, Phys. Rev. Lett. 78, 1335-1338 (1997).

[20] J.B. Miller, D. M. Zumbühl, C. M. Marcus, Y. B. Lyanda- Geller, D. GoldhaberGordon, K. Campman, and a. C. Gossard, Phys. Rev. Lett. 90, 076807 (2003).

[21] A.D. Caviglia, M. Gabay, S. Gariglio, N. Reyren, C. Cancel- lieri, and J.-M. Triscone, Phys. Rev. Lett. 104, 126803 (2010).

[22] H. Nakamura, T. Koga, and T. Kimura, Phys. Rev. Lett. 108, 206601 (2012). 
[23] Z. Zhong, A. Tóth, and K. Held, Phys. Rev. B 87, 161102 (2013).

[24] Y. H. Park, H.-J. Kim, J. Chang, S. H. Han, J. Eom, H.-J. Choi, and H. C. Koo, Appl. Phys. Lett. 103, 252407 (2013).

[25] S. LaShell, B. McDougall, and E. Jensen, Phys. Rev. Lett. 77, 3419-3422 (1996).

[26] D. Popović, F. Reinert, S. Hüfner, V. Grigoryan, M. Springborg, H. Cercellier, Y. Fagot-Revurat, B. Kierren, and D. Malterre, Phys. Rev. B 72, 045419 (2005).

[27] I. Gierz, B. Stadtmüller, J. Vuorinen, M. Lindroos, F. Meier, J. Hugo Dil, K. Kern, and C. R. Ast, Phys. Rev. B 81, 245430 (2010).

[28] Yu. Koroteev, G. Bihlmayer, J. Gayone, E. Chulkov, Stefan Blügel, P. Echenique, and Ph. Hofmann, Phys. Rev. Lett. 93, 046403 (2004).

[29] C. Ast, J. Henk, A. Ernst, L. Moreschini, M. Falub, D. Pacilé, P. Bruno, K. Kern, and M. Grioni, Phys. Rev. Lett. 98, 186807 (2007).

[30] L. Moreschini, A. Bendounan, H. Bentmann, M. Assig, K. Kern, F. Reinert, J. Henk, C. Ast, and M. Grioni, Phys. Rev. B 80, 035438 (2009).

[31] O. Krupin, G. Bihlmayer, K. Starke, S. Gorovikov, J. Prieto, K. Döbrich, Stefan Blügel, and G. Kaindl, Phys. Rev. B 71, 201403 (2005).

[32] A.M. Shikin, A. G. Rybkin, D. E. Marchenko, D. Yu. Usa- chov, V. K. Adamchuk, A. Yu. Varykhalov, and O. Rader, Physics of the Solid State 52, 1515-1525 (2010).

[33] S. Mathias, A. Ruffing, F. Deicke, M. Wiesenmayer, I. Sakar, G. Bihlmayer, E. V. Chulkov, Yu. M. Koroteev, P. M. Echenique, M. Bauer, and M. Aeschlimann, Phys. Rev. Lett. 104, 066802 (2010).

[34] A.G. Rybkin, A. M. Shikin, V. K. Adamchuk, D. Marchenko, C. Biswas, A. Varykhalov, and O. Rader, Phys. Rev. B 82, 233403 (2010).

[35] G. Bihlmayer, Yu. M. Koroteev, P. M. Echenique, E. V. Chulkov, and S. Blügel, Surface Science 600, 3888 (2006).

[36] G. Bihlmayer, S. Blügel, and E. V. Chulkov, Phys. Rev. B 75, 195414 (2007).

[37] H. Bentmann, T. Kuzumaki, G. Bihlmayer, S. Blügel, E. V. Chulkov, F. Reinert, and K. Sakamoto, Phys. Rev. B 84, 115426 (2011).

[38] O. Krupin, G. Bihlmayer, K. M. Döbrich, J. E. Prieto, K. Starke, S. Gorovikov, S. Blügel, S. Kevan, and G. Kaindl, New Journal of Physics 11, 013035 (2009).

[39] J.-H. Park, C. H. Kim, H.-W. Lee, and J. H. Han, Phys. Rev. B 87, 041301 (2013).

[40] P. M. Haney, H.-W. Lee, K.-J. Lee, A. Manchon, and M. D. Stiles, Phys. Rev. B 88, 214417 (2013).

[41] S. Grytsyuk, A. Belabbes, P. M. Haney, H.-W. Lee, K.-J. Lee, M. D. Stiles, U. Schwingenschlögl, and A. Manchon, Phys. Rev. B 93, 174421 (2016).

[42] M.Z. Hasan, and C. Kane, Rev. Mod. Phys. 82, 3045 (2010).

[43] X.L. Qi, and S.-C. Zhang, Rev. Mod. Phys. 83, 1057-1110 (2011).

[44] T.O. Wehling, A.M. Black-Schaffer, and A.V. Balatsky, Advances in Physics 63 , 1 (2014)

[45] Y. Ando, and L. Fu, Annual Review of Condensed Matter Physics 6, 361 (2015).

[46] Y.L. Chen, J.G. Analytis, J.-H. Chu, Z.K. Liu, S.-K. Mo, X.L. Qi, H. J. Zhang, D. H. Lu, X. Dai, Z. Fang, S. C. Zhang, I. R. Fisher, Z. Hussain, and Z.-X. Shen, Science 325, 178 (2009).

[47] H. Zhang, C.-X. Liu, X.-L. Qi, X. Dai, Z. Fang, and S.-C. Zhang, Nature Physics 5, 438-442 (2009). 
[48] P. Gehring, H. M. Benia, Y. Weng, R. Dinnebier, C. R. Ast, M. Burghard, and K. Kern, Nano letters 13, 1179 (2013).

[49] E.L. Ivchenko and G.E. Pikus, JETP Lett. 27, 604 (1978).

[50] V. M. Edelstein, Solid State Commun. 73, 233-235 (1990).

[51] A. Chernyshov, M. Overby, X. Liu, J. K. Furdyna, Y. Lyanda-Geller, and L. P. Rokhinson, Nature Phys. 5, 656 (2009).

[52] A. Manchon and S. Zhang, Phys. Rev. B 79, 094422 (2009).

[53] A. Matos-Abiague, R. L. Rodríguez-Suárez, Phys. Rev. B 80, 094424 (2009).

[54] S. G. Tan, M. B. A. Jalil, X.-J. Liu, and T. Fujita, Annals of Physics, vol. 326, 207 (2011).

[55] E. van der Bijl and R. A. Duine, Phys. Rev. B 86, 094406 (2012).

[56] X. Wang, and A. Manchon, Phys. Rev. Lett. 108, 117201 (2012); X. Wang, C.O. Pauyac and A. Manchon, Phys. Rev. B 89, 054405 (2014).

[57] D. A. Pesin and A. H. MacDonald, Phys. Rev. B 86, 014416 (2012).

[58] H. Li, H. Gao, L. P. Zârbo, K. Výborný, X. Wang, I. Garate, F. Dogăn, A. Cějchan, J. Sinova, T. Jungwirth, and A. Manchon, Phys. Rev. B 91, 134402 (2015).

[59] K.-S. Lee, D. Go, A. Manchon, P. M. Haney, M. D. Stiles, H.-W. Lee, and K.-J. Lee, Phys. Rev. B 91, 144401 (2015).

[60] A. Qaiumzadeh, R. A. Duine, and M. Titov, Phys. Rev. B 92, 014402 (2015).

[61] K. Obata and G. Tatara, Phys. Rev. B 77, 214429 (2008).

[62] K.-W. Kim, S.-M. Seo, J. Ryu, K.-J. Lee, and H.-W. Lee, Phys. Rev. B 85, 180404(R) (2012).

[63] J. Ryu, S.-M. Seo, K.-J. Lee, and H.-W. Lee, J. Magn. Magn. Mater. 324, 1449 (2012).

[64] J. Linder, Phys. Rev. B 87, 054434 (2013).

[65] J. Linder and M. Alidoust, Phys. Rev. B 88, 064420 (2013).

[66] K. M. D. Hals and A. Brataas, Phys. Rev. B 88, 085423 (2013).

[67] K. M. D. Hals and A. Brataas, Phys. Rev. B 89, 064426 (2014).

[68] M. Stier, R. Egger, and M. Thorwart, Phys. Rev. B 87, 184415 (2013).

[69] M. Stier, M. Creutzburg, and M. Thorwart, Phys. Rev. B 90, 014433 (2014).

[70] M. Stier, and M. Thorwart, Phys. Rev. B 92, 220406(R) (2015).

[71] M. D'yakonov and V. Perel, JETP Letters 13, 657 (1971).

[72] H.B.M. Saidaoui and A. Manchon, Phys. Rev. Lett. 117, 036601 (2016).

[73] S. Zhang, P.M. Levy and A. Fert, Phys. Rev. Lett. 88, 236601 (2002).

[74] S. Zhang, and Z. Li, Phys. Rev. Lett. 93, 127204 (2004).

[75] H. Kurebayashi, J. Sinova, D. Fang, A. C. Irvine, J. Wunderlich, V. Novak, R. P. Campion, B. L. Gallagher, E. K. Vehstedt, L. P. Zârbo, K. Vyborny, A. J. Ferguson, and T. Jungwirth, Nature Nanotech. 9, 211 (2014).

[76] F. Freimuth, S. Blügel, and Y. Mokrousov, Phys. Rev. B 90, 174423 (2014).

[77] V. P. Amin, and M. D. Stiles, Phys. Rev. B 94, 104419 (2016).

[78] J. Wunderlich, A. C. Irvine, Jairo Sinova, B. G. Park, L. P. Zârbo, X. L. Xu, B. Kaestner, V. Novák and T. Jungwirth, Nature Physics 5, 675 (2009).

[79] M. D'yakonov and V. Perel, Soviet Physics Solid State 13, 3023 (1972). 
[80] K.-W. Kim, J.-H. Moon, K.-J. Lee, and H.-W. Lee, Phys. Rev. Lett. 108, 217202 (2012).

[81] K.-W. Kim, H.-W. Lee, K.-J. Lee, and M.D. Stiles, Phys. Rev. Lett. 111, 216601 (2013).

[82] A. Thiaville, S. Rohart, E. Jué, V. Cros and A. Fert, EuroPhys. Lett. 100, 57002 (2012).

[83] M. Endo, F. Matsukura, and H. Ohno, Appl. Phys. Lett. 97, 222501 (2010).

[84] D. Fang, H. Kurebayashi, J. Wunderlich, K. Výborný, L. P. Zârbo, R. P. Campion, A. Casiraghi, B. L. Gallagher, T. Jungwirth, and A. J. Ferguson, Nature Nanotech. 6, 413 (2011).

[85] B. A. Bernevig and O. Vafek, Phys. Rev. B 72, 033203 (2005).

[86] I. Garate and H. A. MacDonald, Phys. Rev. B. 80, 134403 (2009).

[87] K. M. D. Hals, A. Brataas and Y. Tserkovnyak, EuroPhys. Lett. 90, 47002 (2010).

[88] J. Nogués and I. K. Schuller, J. Magn. Magn. Mater. 192, 203-232 (1999).

[89] T. Giamarchi, C. Rüegg, and O. Tchernyshov, Nature Physics 4, 198 (2008).

[90] A. S. Nuñez, R. A. Duine, P. Haney, and A. H. MacDonald, Phys. Rev. B 73, 214426 (2006).

[91] A. H. MacDonald and M. Tsoi, Philos. Trans. R. Soc. A Math. Phys. Eng. Sci. 369, 3098 (2011).

[92] E. V. Gomonay and V. M. Loktev, Low Temp. Phys. 40, 17 (2014).

[93] P. M. Haney and A. H. MacDonald, Phys. Rev. Lett. 100, 196801 (2008).

[94] H. Reichlova, D. Kriegner, V. Holý, K. Olejník, V. Novak, M. Yamada, K. Miura, S. Ogawa, H. Takahashi, T. Jungwirth, and J. Wunderlich, Phys. Rev. B 92, 165424 (2015).

[95] A. Manchon, arXiv:1608.00140v1

[96] J.Železný, H. Gao, K. Výborný, J. Zemen, J. Mašek, A. Manchon, J. Wunderlich, J. Sinova, and T. Jungwirth, Phys. Rev. Lett. 113, 157201 (2014).

[97] J.Železný, H. Gao, Aurélien Manchon, Frank Freimuth, Yuriy Mokrousov, J. Zemen, J. Mašek, Jairo Sinova, T. Jungwirth, arXiv:1604.07590 (2016).

[98] T. Jungwirth, X. Martí, P. Wadley and J. Wunderlich, Nature Nanotechnology 11, $231(2016)$.

[99] V. Baltz, A. Manchon, M. Tsoi, T. Moriyama, T. Ono, and Y. Tserkovnyak, arXiv:1606.04284.

[100] A. Dýrdal and J. Barnas, Phys. Rev. B 92, 165404 (2015).

[101] H. Li, X. Wang, and A. Manchon, Phys. Rev. B 93, 035417 (2016).

[102] H. Li, and A. Manchon, Phys. Rev. B 93, 235317 (2016).

[103] C. Gould, C. Rüster, T. Jungwirth, E. Girgis, G.M. Schott, R. Giraud, K. Brunner, G. Schmidt, and L.W. Molenkamp, Phys. Rev. Lett. 93, 117203 (2004).

[104] , J. Moser, A. Matos-Abiague, D. Schuh, W. Wegscheider, J. Fabian, and D. Weiss, Phys. Rev. Lett. 99, 056601 (2007).

[105] B. G. Park, J. Wunderlich, D. A. Williams, S. J. Joo, K.Y. Jung, K. H. Shin, K. Olejník, A. B. Shick, and T. Jungwirth, Phys. Rev. Lett. 100, 087204 (2008).

[106] A. Manchon, Phys. Rev. B 83, 172403 (2011).

[107] F. Mahfouzi, N. Nagaosa, and B. K. Nikolić, Phys. Rev. Lett. 109, 166602 (2012).

[108] M. Z. Hasan and C. L. Kane, Rev. Mod. Phys. 82, 3045 (2010). 
[109] A. R. Mellnik, J. S. Lee, A. Richardella, J. L. Grab, P. J. Mintun, M. H. Fischer, A. Vaezi, A. Manchon, E.-A. Kim, N. Samarth, and D. C. Ralph, Nature 511, 449 (2014),

[110] Y. Fan, P. Upadhyaya, X. Kou, M. Lang, S. Takei, Z.Wang, J. Tang, L. He, L.-t. Chang, M. Montazeri, G. Yu, W. Jiang, T. Nie, R. N. Schwartz, Y. Tserkovnyak, and K. L. Wang, Nat. Mater. 13, 699 (2014).

[111] Y. Fan, X. Kou, P. Upadhyaya, Q. Shao, L. Pan, M. Lang, X. Che, J. Tang, M. Montazeri, K. Murata, L.-T. Chang, M. Akyol, G. Yu, T. Nie, K. L. Wong, J. Liu, Y. Wang, Y. Tserkovnyak, and K. L. Wang, Nat. Nanotechnol. 11, 352 (2016).

[112] Y. Wang, P. Deorani, K. Banerjee, N. Koirala, M. Brahlek, S. Oh, and H. Yang, Phys. Rev. Lett. 114, 257202 (2015).

[113] X.-L. Qi, T. L. Hughes, and S.-C. Zhang, Phys. Rev. B 78, 195424 (2008).

[114] I. Garate and M. Franz, Phys. Rev. Lett. 104, 146802 (2010).

[115] C.-Z. Chang, et al., Science 340, 167 (2013); X. Kou et al., Phys. Rev. Lett. 113, 137201 (2014)

[116] T. Yokoyama, Phys. Rev. B 81, 241410(R) (2010).

[117] Y. Tserkovnyak and D. Loss, Phys. Rev. Lett. 108, 187201 (2012).

[118] K. Nomura and N. Nagaosa, Phys. Rev. B 82, 161401(R) (2010).

[119] Y. Tserkovnyak, D. A. Pesin, and D. Loss, Phys. Rev. B 91, 041121(R) (2015).

[120] M. H. Fischer, A. Vaezi, A. Manchon, and E.-A. Kim, Phys. Rev. B 93, 125303 (2016).

[121] Junji Fujimoto, Akio Sakai, and Hiroshi Kohno, Phys. Rev. B 87, 085437 (2013).

[122] A. Sakai and H. Kohno, Phys. Rev. B 89, 165307 (2014).

[123] K. Taguchi, K. Shintani, and Y. Tanaka, Phys. Rev. B 92, 035425 (2015).

[124] F. Mahfouzi, N. Nagaosa, and B. K. Nikolić, Phys. Rev. B 90, 115432 (2014).

[125] P.-H. Chang, T. Markussen, S. Smidstrup, K. Stokbro, and B. K. Nikolić, Phys. Rev. B 92, 201406(R) (2015).

[126] F. Mahfouzi, B. K. Nikolić, and N. Kioussis Phys. Rev. B 93, 115419 (2016).

[127] P. B. Ndiaye, C. A. Akosa, M. H. Fischer, A. Vaezi, E-A. Kim, and A. Manchon, arxiv: $1509.06929 \mathrm{v} 1$

[128] A. Manchon and H. Yang, Chapter 10. Spin-Orbit Torques: Experiments and Theory, in "Spintronics Handbook: Spin Transport and Magnetism", Second Edition, Edited by E.Y. Tsymbal and I. Zutić, CRC Press (2017).

[129] S. Monso, B. Rodmacq, S. Auffret, G. Casali, F. Fettar, B. Gilles, B. Dieny, and P. Boyer, Appl. Phys. Lett. 80, 4157 (2002).

[130] A. Manchon, C. Ducruet, L. Lombard, S. Auffret, B. Rodmacq, B. Dieny, S. Pizzini, J. Vogel, V. Uhlír, M. Hochstrasser, and G. Panaccione, J. Appl. Phys. 104, 043914 (2008).

[131] S. Emori, T. Nan, A. M. Belkessam, X. Wang, A. D. Matyushov, C. J. Babroski, Y. Gao, H. Lin, and N. X. Sun, Phys. Rev. B 93, 180402(R) (2016).

[132] X. Qiu et al., Nat. Nanotechnol. 10, 333-338 (2015).

[133] K. M. D. Hals and A. Brataas, Phys. Rev. B 87, 174409 (2013);

[134] C. Ciccarelli, L. Anderson,V. Tshitoyan, A. J. Ferguson, F. Gerhard, C. Gould, L.W. Molenkamp, J. Gayles, J. Železný, L. Šmejkal, Z. Yuan, J. Sinova, F. 
Freimuth and T. Jungwirth, Nat. Phys. 12, 855 (2016).

[135] A. Ohtomo and H. Hwang, Nature (London) 427, 423 (2004).

[136] P. Zubko, S. Gariglio, M. Gabay, P. Ghosez, and J.-M. Triscone, Annu. Rev. Condens. Matter Phys. 2, 141 (2011).

[137] H. Y. Hwang, Y. Iwasa, M. Kawasaki, B. Keimer, N. Nagaosa, and Y. Tokura, Nature Materials 11, 103 (2012).

[138] A. Brinkman, M. Huijben, M. van Zalk, J. Huijben, U. Zeitler, J. C. Maan, W. G. van der Wiel, G. Rijnders, D. H. A. Blank, and H. Hilgenkamp, Nat.Mater. 6 , $493(2007)$.

[139] D. A. Dikin, M. Mehta, C. W. Bark, C. M. Folkman, C. B. Eom, and V. Chandrasekhar, Phys.Rev.Lett. 107, 056802 (2011).

[140] Lu Li, C. Richter, J. Mannhart, and R. C. Ashoori, Nat. Phys. 7, 762 (2011).

[141] N. Reyren, M. Bibes, E. Lesne, J.-M. George, C. Deranlot, S. Collin, A. Barthélémy, and H. Jaffrès, Phys. Rev. Lett. 108, 186802 (2012).

[142] W. Han, X. Jiang, A. Kajdos, S.-H. Yang, S. Stemmer and S. S. P. Parkin, Nat. Comm. 4, 2134 (2013).

[143] K. Narayanapillai, K. Gopinadhan, X. Qiu, A. Annadi, Ariando, T. Venkatesan, and H. Yang, Appl. Phys. Lett. 105, 162405 (2014). 